\title{
LA BENEFICENCIA DEL MÉDICO EN EL TRATAMIENTO DEL ENFERMO CON CÁNCER TERMINAL DESDE LA EXPERIENCIA DEL FAMILIAR
}

\author{
Norma Alicia Ordóñez Vázquez ${ }^{1}$, Mariana Becerra Sánchez ${ }^{2}$, Zuraya Monroy Nasr²
}

Resumen: El objetivo de este artículo es conocer, a partir de la experiencia de familiares de enfermos con cáncer terminal, la beneficencia del médico hacia el enfermo. Este propósito es parte de un trabajo más amplio titulado "Los cuidados, la atención y la decisión de los enfermos con cáncer terminal al final de la vida: una visión desde la experiencia del familiar”. La experiencia de los familiares proporciona información acerca del actuar del médico sobre los riesgos y beneficios de tratamientos o procedimientos. Se llevó a cabo un estudio cualitativo que parte del paradigma hermenéutico fenomenológico, elegido porque permite conocer la percepción que tienen las personas del mundo en el cual viven y el significado que le dan. Se entrevistó a 11 familiares y cuidadores primarios y se utilizó el análisis temático y el método de van Manen. La experiencia de los familiares muestra que algunos médicos, en ocasiones, no actuaron a partir del principio de beneficencia, porque no proporcionaron información detallada, la omitieron o no hubo comunicación, no respetaron las decisiones y no controlaron o eliminaron el malestar o dolor del enfermo. Los médicos deben reconocer y subsanar situaciones en las que no logran cumplir con este principio.

Palabras clave: beneficencia, cáncer, fenomenología hermenéutica, análisis temático, método de van Manen, experiencia de familiares

\section{Beneficence of the doctor in the treatment of patients with terminal cancer from the experience of family}

Abstract: The aim of this paper is to learn from the experience of relatives of patients with terminal cancer about the medical beneficence during a terminal illness. This paper is part of a broader study entitled "The care, attention and decision of patients with terminal cancer at the end of life: a vision from the experience of family". The experience of the family provides information about the act from the doctor about the risks and benefits of treatments or procedures. A qualitative study was carried out that part of the phenomenological hermeneutical paradigm. It is approached from the phenomenological stance because it lets you know the perception that people have of the world in which they live and the meaning they give you. 11 family members and primary caregivers, were interviewed. Thematic analysis and the method of van Manen were used. The experience of family shows that some doctors sometimes did not act from principle of beneficence because they did not provide detailed information, omitted or no communication, they did not respect their decisions and not controlled or eliminated the discomfort or pain of the patient. Physicians must recognize and address situations that fail to comply with this principle.

Key words: beneficence, cancer, phenomenology hermeneutics, thematic analysis, method of van Manen, experience of family

A beneficência do médico no tratamento do paciente com câncer terminal, a partir da experiência familiar

Resumo: $\mathrm{O}$ objetivo deste artigo é conhecer, a partir da experiência de familiares de pacientes com câncer terminal, a beneficência do médico ao paciente. Este objetivo faz parte de um trabalho mais amplo intitulado "Os cuidados, a atenção e a decisão de pacientes com câncer terminal no final da vida: uma visão da experiência da família". A experiência dos membros da família fornece informaçôes sobre a atuação do médico sobre os riscos e benefícios de tratamentos ou procedimentos. Um estudo qualitativo foi conduzido partindo do paradigma hermenêutico fenomenológico, escolhido porque este método permite conhecer a percepção que têm as pessoas no mundo em que vivem e o significado que elas o dáo. Foram entrevistados 11 familiares e cuidadores primários e foi utilizada a análise temática e o método de van Manen. A experiência dos familiares mostra que alguns médicos em certas ocasióes não agiram a partir do princípio da beneficência, porque não forneceram informaçôes detalhadas, omitiram-na ou não havia nenhuma comunicação, não respeitaram as decisóes e não controlaram ou eliminaram o desconforto ou dor do paciente. Os médicos devem reconhecer e corrigir situaçóes que não respeitam este princípio.

Palavras-chave: beneficiência, cáncer, fenomenología hermenêutica, análise temática, método de van Manen, experiência defamiliares

\footnotetext{
${ }^{1}$ Facultad de Medicina, Universidad Nacional Autónoma de México, México

Correspondencia: camel1806@yahoo.com.mx

${ }^{2}$ Facultad de Psicología, Universidad Nacional Autónoma de México, México.
} 


\section{Introducción}

El cáncer es una enfermedad que en los últimos años ha registrado un incremento en su incidencia. En México, en 2013, ocupó la tercera causa de muerte(1). En la mayoría de los casos, quienes se hacen responsables del cuidado de los enfermos son los familiares. Algunos fungen como cuidadores; sin embargo, tanto cuidadores como familiares aportan datos relevantes acerca del proceso por el que atraviesa el enfermo, como por ejemplo la información proporcionada por el médico, el tratamiento elegido y la toma de decisiones al final de la vida.

Los enfermos con cáncer terminal, al presentar diversos síntomas, requieren ser atendidos en primera instancia por su médico tratante o ser canalizados con otros especialistas. Necesitan recibir información acerca de su diagnóstico y pronóstico, y las diferentes opciones de medicamentos o tratamientos a seguir.

Actualmente, la autonomía de la que goza el paciente le otorga el derecho de elegir lo que desea o no en su tratamiento médico, y la información que desea recibir. Esto ha permitido que el enfermo tenga una participación más activa en la relación con su médico y en la toma de decisiones.

De acuerdo con Cataldi(2), durante 25 siglos el médico actuó y creyó que su deber profesional era hacer el bien, aun en contra de la voluntad del paciente. Hacer el bien y evitar el mal, sin considerar la opinión, el deseo o la voluntad del enfermo, fue el paradigma clásico. El médico no solo tenía el conocimiento necesario y creía saber lo que le convenía o no al paciente, sino que además era el único capacitado para tomar decisiones.

El Informe Belmont hizo públicos principios basados en la bioética, que se formularon para proteger a los seres humanos de cualquier violación al participar en algún tratamiento o investigación. Posteriormente, estos fueron ampliados y aplicados a la ética biomédica por Beauchamp y Childress(3). De acuerdo con estos autores, estos principios son beneficencia, no maleficencia, autonomía y justicia.

El principio de beneficencia (principio capital de la ética hipocrática) es un valor moral esencial, pues constituye un prerrequisito de toda buena práctica. Actuar siempre para hacer el bien, tomando en cuenta el beneficio que le reportarán al enfermo las acciones médicas, es el principio básico de la disciplina médica(4).

En años recientes, el médico debe atender siempre tres deberes como mínimo: hacer lo que sea mejor para su paciente, tener en cuenta los intereses de la sociedad y respetar la autonomía del paciente.

- Médico: guiado por el principio de beneficencia.

- Paciente: guiado por el principio de autonomía.

- Sociedad: guiada por el principio de justi$\operatorname{cia}(5,2)$.

Los principios bioéticos cambiaron la visión de beneficencia paternalista, en la que el médico tomaba las decisiones por el bien del paciente. Ahora el paciente puede tomar sus decisiones de acuerdo con sus valores, creencias y planes de vida.

Acorde con el principio bioético de beneficencia, el médico debe dirigir sus acciones en busca del beneficio del paciente y de la sociedad. Evitar cualquier acción que pueda dañar al paciente y exponerlo al mínimo riesgo. Promover y mantener una relación médico-paciente amable, de confianza mutua y con comunicación permanente en ambos sentidos. El médico tiene el compromiso de proporcionar información al paciente y a sus familiares, con oportunidad, en forma completa, comprensible para ellos y con veracidad; que incluya los diagnósticos de probabilidad o certeza, el programa de estudios y tratamientos; sus objetivos, los riesgos, efectos adversos, secuelas y complicaciones que se podrían presentar; los beneficios esperados, el pronóstico, sus variaciones y las expectativas de curación, control, mejoría o paliación de la enfermedad. Cuando la información no es suficiente o clara, el paciente tiene derecho a hacer las preguntas que considere necesarias y a que se le respondan. Al final de la atención o cuando se requiera transferir al paciente a otro hospital, el médico debe entregar un informe completo y por escrito de la atención otorgada(6). 
En el caso de los pacientes con cáncer terminal, es importante que este principio sea aplicado de la mejor manera, pues la evolución de la propia enfermedad lleva a tomar decisiones vitales para mejorar su calidad de vida. En estos pacientes, lo mejor que puede hacer el médico cuando ya no hay tratamiento es, como lo menciona su código ético, curar, o aliviar cuando no se puede curar y siempre apoyar y acompañar al paciente(5).

El principal objetivo de este artículo es conocer, desde la experiencia de los familiares del enfermo con cáncer terminal, la beneficencia del médico hacia el paciente, es decir, la información que se proporciona acerca de los riesgos y beneficios de tratamientos o procedimientos, la comunicación de diagnósticos y pronósticos y la relación médico-paciente que se establece.

\section{Método}

\section{Tipo de investigación}

Se trata de una investigación de corte cualitativo que parte del paradigma hermenéutico fenomenológico. La fenomenología hermenéutica es una aproximación cualitativa interpretativa, por medio de la cual se puede estudiar y comprender las experiencias diarias de las personas(7), en este caso, de los familiares del enfermo terminal. Esta perspectiva teórica, empleada en diversos estudios en el área de la salud, busca interpretar y entender estas experiencias, y permite al investigador descubrir los significados ocultos o que se dan por conocidos, es decir, aquello inadvertido por los sujetos en su vida diaria(8).

La fenomenología es considerada una disciplina filosófica y un método de investigación(9). Desde esta perspectiva se interpretó y analizó la experiencia de la enfermedad en familiares de enfermos terminales de cáncer. Es una mirada desde la cual se han realizado diversos estudios en el área de la salud —en enfermería, medicina, trabajo social, entre otras-, que se han enfocado en identificar la vivencia o experiencia de las personas respecto del dolor, la enfermedad, los cuidados en los servicios de salud, la relación de profesionales de la salud con pacientes y familiares, entre $\operatorname{otros}(10,11,8,12-15)$.

\section{Participantes}

Se entrevistó a cuidadores primarios (familiares que se hicieron responsables de todo el cuidado del paciente a lo largo de su enfermedad) y familiares (miembros de la familia que no participaron de forma activa en el cuidado) de enfermos con cáncer terminal que fueron atendidos en hospitales de la ciudad de México. Cinco enfermos fueron atendidos en hospitales públicos, uno en un centro privado y después en uno público, y otro enfermo solo en hospital privado.

Se utilizó un muestreo por conveniencia, que trata de muestras integradas por informantes cautivos o por voluntarios, y depende de la accesibilidad que se tenga para entrevistarlos(16).

\section{Instrumento}

Se empleó una guía de entrevista semiestructurada, conformada por cinco ejes (tabla 1), desde los que se exploró la experiencia de cuidadores y familiares del enfermo en este transitar de la enfermedad en el hospital, con los médicos, y con las decisiones al final de la vida. Los criterios para la elaboración de la guía se basaron en los objetivos de la investigación y en la revisión bibliográfica consultada para realizar el marco teórico. Los resultados que se presentan en este artículo se desprenden de uno de los varios ejes temáticos investigados. En este artículo se explora el eje 4. Relación médico paciente. ¿Realmente se aplica el principio de beneficencia?

Tabla 1. Ejes temáticos de la guía de entrevista semiestructurada.

1. El inicio de la enfermedad, darse cuenta de ésta.

2. Principales necesidades durante la enfermedad.

3. El tránsito por los servicios de salud y la atención del médico.

4. Relación médico paciente. ¿Realmente se aplica el principio de beneficencia?

5. Las decisiones para tener una muerte digna, preparación para ésta. 


\section{Procedimiento}

Se realizaron 11 entrevistas, de las cuales ocho formaron díadas cuidador/familiar. Las tres restantes solamente se realizaron a cuidadores, ya que por diversas cuestiones personales de los participantes no se pudo contactar a otro familiar para llevar a cabo la entrevista y así completar las díadas. El principal objetivo de formar díadas fue para complementar la información que se obtuvo del cuidador primario.

Seis entrevistas se llevaron a cabo en el espacio de una institución especializada en la atención del cáncer y cinco en espacios públicos, como restaurantes o cafeterías. Se obtuvo consentimiento informado para realizar las entrevistas y audiograbarlas. Se explicó a los participantes los objetivos del estudio y se aclaró que el participar o negarse a hacerlo no afectaría la atención o tratamientos que recibía su familiar enfermo o él en el hospital. Además, se solicitó a los participantes un seudónimo con el que serían nombrados en el estudio. Aunque algunos prefirieron no elegirlo, sus nombres fueron cambiados para conservar el anonimato. Este trabajo fue aprobado por las comisiones de Investigación y Ética de la institución especializada en la atención del cáncer.

Después de transcribir las entrevistas, se utilizó el análisis temático y el método de van Manen (utilizado en la fenomenología hermenéutica) para el análisis de las entrevistas. Esto se logró mediante la actividad reflexiva de la escritura y lectura, como un medio de transmitir la esencia de la experiencia, y consiste en comparar la grabación con la transcripción para asegurar la exactitud. Se extrajeron temas y subtemas de cada entrevista (tabla 2) y se obtuvo las experiencias de cada participante, tratando de comprender e interpretar el significado de esta(7). En este artículo se presentan los resultados del subtema 6 . Beneficencia del médico en el tratamiento.
Tabla 2. Temas y subtemas.

\begin{tabular}{|l|l|}
\hline Tema & Subtema \\
\hline $\begin{array}{l}\text { 1. Experiencia del proceso } \\
\text { de enfermedad }\end{array}$ & $\begin{array}{l}\text { 1. Inicio de la enfermedad } \\
\text { 2. Tratamiento } \\
\text { 3. Evolución de la } \\
\text { enfermedad }\end{array}$ \\
\hline $\begin{array}{l}\text { 2. Cuidados y atención } \\
\text { a las necesidades del } \\
\text { enfermo }\end{array}$ & $\begin{array}{l}\text { 4. Cuidados paliativos: } \\
\text { necesidades médicas, } \\
\text { psicológicas, sociales } \\
\text { y espirituales de los } \\
\text { enfermos } \\
\text { 5. El médico como apoyo } \\
\text { al enfermo y a la familia }\end{array}$ \\
\hline $\begin{array}{l}\text { 3. Relación médico } \\
\text { paciente }\end{array}$ & $\begin{array}{l}\text { 6. Beneficencia del } \\
\text { médico en el tratamiento } \\
\text { 7. Adecuada } \\
\text { comunicación }\end{array}$ \\
\hline $\begin{array}{l}\text { 4. Decisiones al final de } \\
\text { la vida }\end{array}$ & $\begin{array}{l}\text { 8. Respeto a la autonomía } \\
\text { 9. El enfermo o la familia } \\
\text { como tomadores de } \\
\text { decisiones }\end{array}$ \\
\hline
\end{tabular}

\section{Resultados}

Se entrevistó a 11 familiares de pacientes con cáncer terminal; seis de los enfermos ya habían muerto y solamente una paciente se encontraba en remisión. En las tablas 3 y 4 se detallan las características de familiares y enfermos.

Tabla 3. Características de familiares de enfermos con cáncer terminal.

\begin{tabular}{|l|}
\hline Familiares \\
\hline 11 participantes \\
8 formaron díadas cuidador/familiar $=$ (4 cuidadores $/ 4$ \\
familiares) \\
3 cuidadores primarios \\
\hline Edad \\
$16-76$ años \\
\hline Escolaridad \\
3 educación básica \\
2 preparatoria \\
6 licenciatura \\
1 posgrado \\
\hline Parentesco con el enfermo \\
4 hijas \\
2 esposos/ 1 esposa \\
1 madre \\
3 hermanas \\
\hline
\end{tabular}


Tabla 4. Características de los enfermos con cáncer terminal.

\begin{tabular}{|l|}
\hline Enfermos \\
\hline 7 enfermos \\
4 enfermos $=4$ cuidadores/4 familiares \\
3 enfermos=3 cuidadores \\
\hline Tipos de cáncer: \\
1 piel basocelular (se encontraba en remisión) \\
2 cáncer de mama \\
1 cáncer de esófago \\
1 tumor cerebral \\
1 leucemia \\
1 ovario \\
\hline Tipo de hospital \\
2 Instituto especializado en atención de cáncer \\
3 Público \\
1 Privado/Instituto especializado en atención de cáncer \\
1 Privado \\
\hline
\end{tabular}

Uno de los principios bioéticos que debe considerarse como pilar es la beneficencia, porque todos los conocimientos que posee el médico deben dirigirse a hacer el bien o beneficiar a los pacientes, defender sus derechos, prevenir el daño, eliminar los riesgos, el malestar y el dolor. La experiencia de los familiares de los enfermos mostró que los médicos, en ocasiones, no actuaron a partir de este principio, porque no proporcionaron información detallada, la omitieron o no les importó controlar o eliminar las condiciones de malestar o dolor del enfermo.

Lola (cuidadora), desde su experiencia, narra la falta de interés del médico para proporcionarle ayuda y atender a las dudas que le expresó sobre los síntomas de su mamá. La actuación del médico ante esta situación no fue la de un profesional que ayuda, dando una solución a las dudas y malestar del paciente, estableciendo una buena comunicación. También se observó que no intentó auxiliar a la paciente porque no la canalizó a otro servicio donde la atendieran y mitigaran su malestar, como a cuidados paliativos. Desde la experiencia de Julia (cuidadora), el médico no actuó desde el principio ético de beneficencia, porque no intentó ayudarla e indicarle los medicamentos que podían aliviar los síntomas de su hermana, y no le dijeron que podía ser tratada en cuidados paliativos. Además, el médico no le proporcionó información a la enferma sobre su pronóstico, sino que todo lo comunicaba a la familia. En se- guida se puede apreciar la experiencia de estas dos participantes.

Lola (cuidadora): Para que no se sienta mal, y yo le decía al doctor, "es que mire, está pasando, está pasando esto", (el médico decía) "no, no, no, eso no sale, eso no tiene nada que ver con el cáncer", este, no..."

... “no, no, no, no, eso no es de aqui de cáncer".. Él nada más su especialidad, eso es algo de las cosas que pasan aqui... ellos no, no ven las otras partes que tiene la persona...

Julia (cuidadora): ... fuimos un día a consulta, ibamos a consulta por el medicamento no por otra cosa, mi her-mana necesitaba unas inyecciones para contro-lar los efectos de la quimio, y el médico nos dice - "no hay" y yo le digo "y entonces, ¿qué hacemos?" y dice "no sé, es su problema", yo le dije no es mi problema, usted es el médico y me está dando un medicamento, y si no lo hay pues tendría que sustituirlo por otro que sí hay o decirme dónde conseguir ese medicamento. "No, ya, ¿para qué?"; mi hermana estaba ahi y asi nos dijo "ya ipara qué?", y ese "ya para que" fue como así.

Después me enteré de que es uno de los mejo-res oncólogos del país. Yo diría que no, que no es ni siquiera es un ser humano, o sea para ser el mejor oncólogo del país tienes que ser un ser humano primero..

A partir de la experiencia de Carlos (cuidador) se aprecia que el médico que atendió a su esposa no le proporcionaba información respecto a su pronóstico. Él fue quien se dirigió al médico para preguntarle sobre su estado y si era necesario aplicarle algo para el dolor. Narra que hasta que se dirigió al médico residente fue cuando obtuvo la información, porque el médico que la trataba no se la proporcionaba.

Si, ya me atreví a preguntar eso porque la situación... sus órganos estaban muy atrofiados. No me lo dijo (el médico tratante) pero yo ya lo preveía, uno de los doctores (residentes) me dijo "su corazón está creciendo por el esfuerzo que hace al no haber sangre", me lo dijeron y lo entendi, podia darle un paro cardiaco a falta de sangre, lo más probable es que le diera un paro cardiaco por su corazón. Entonces, al preguntarle si iba a haber complicaciones dolorosas, al doctor, yo pre- 
viendo quisiera que le pusiera algo fuerte, morfina o una droga fuerte, dijo, "no, tal vez no la necesitemos, ni la tenemos aqui en un momento dado, pero espero que no la necesitemos" (Carlos, cuidador).

También se puede ver que, en otros casos, algunos médicos actuaron de acuerdo al principio de beneficencia, ayudando a los enfermos. Desde la experiencia de Celia (cuidadora), el médico que atendió a su hija en un hospital privado, y posteriormente en el hospital especializado en la atención del cáncer, una vez que su seguro de gastos médicos se agotó le proporcionó ayuda y le consiguió la donación de la quimioterapia que estaba recibiendo en el hospital privado, y que era muy costosa. El médico tuvo una buena relación con su hija, que era su paciente. Esta acción por parte del médico muestra el interés y el apoyo por beneficiar a su paciente y así lograr su adecuada recuperación. En el caso de Lucía (cuidadora), el médico, preocupado por la salud de su esposo, lo convence de seguir con la quimioterapia para que alcance una mejor calidad de vida. La relación que estableció con el enfermo y su familia fue buena. Constantemente comunicaba cuáles serían los medicamentos que se le administrarían y los efectos adversos que provocarían, y aclaraba dudas y les daba consejos.

Celia (cuidadora): ... ella se atendió en el hospital privado, pero se fue millón y medio como en un año tres meses, se fue millón y medio, entonces el doctor le llegó a decir que no se preocupara, que si se le terminaba su seguro de gastos mayores, si se atendia aqui en el instituto especializado en la atención del cáncer, que no se preocupara, que ella no estaba para preocuparse ahorita: "no te preocupes Elisa, si se te acaba el seguro, te vas al hospital, ahi te puedo atender también..."

Se le acabó el seguro y se vino para acá, y la verdad es que el doctor le dijo: "Elisa, voy a tratar de que un laboratorio me consiga la quimio que te ponían alla", dice, "que me lo donen, a ver si me lo donan". Y si se lo donaron al doctor y le ponía la misma quimio que le ponian allá...

Lucía (cuidadora): ... y el doctor habla con él, se encierra y le dice: "por favor vuelve a la quimio, puedes vivir más tiempo y con más calidad con quimio", y después de que habia tomado la decisión, habian pasado dos meses sin quimio, pues lo convenció el doctor, por la ganas de vivir.

...te llegas a encariñar con los doctores, de veras, que cómprale algo al doctor en navidad, yo les llevaba dulces. La verdad nos fue muy bien...

El médico siempre nos decía paso a paso todo lo que iba ir haciendo.

En el caso de Refugio (cuidadora), se puede interpretar que los médicos que atendieron a su hermana Lulú procuraron su bienestar, previniendo el dolor y el daño al diagnosticarla y sugerirle que se realizara una operación para remover el tumor en el cerebro. Sin embargo, surgieron complicaciones en la cirugía y no se logró eliminar las condiciones que le generaron riesgo y malestar.

"y este"... le pregunté al doctor dígame sinceramente, "esta operación, ¿la puede salvar?, porque nada más la vamos a hacer sufrir, y el doctor "no, con esta operación ella va a quedar bien", "y si fuera usted su familiar, ¿usted firmaba?", "si, yo firmaba".. firmé... y estuvo en terapia intensiva y la volvieron a operar, pero para mi que fue un tiempo largo... (Refugio, cuidadora).

La beneficencia es uno de los principios esenciales orientados a prevenir el daño y proteger a los pacientes del malestar, como se observó en algunas de las experiencias que se muestran en este trabajo. A pesar de ello, en ocasiones, los médicos que atendieron a los enfermos no lograron cumplir con este principio, ya sea por falta de empatía (beneficencia anulada por factores internos, como insensibilidad, falta de compromiso ético, competencia profesional, honestidad con los pacientes, confidencialidad, mantener buenas relaciones con los pacientes, mejorar la calidad de la atención, responsabilidad profesional), los riesgos de las cirugías (beneficencia reducida por el principio del doble efecto), las limitaciones de tiempo o recursos (principio de justicia) (figura 1). Esto ocasionó que los pacientes fueran los principales perjudicados y sufrieran dolor físico o emocional en el proceso de enfermedad. 
Figura 1. Factores que pueden reducir la beneficencia del médico.
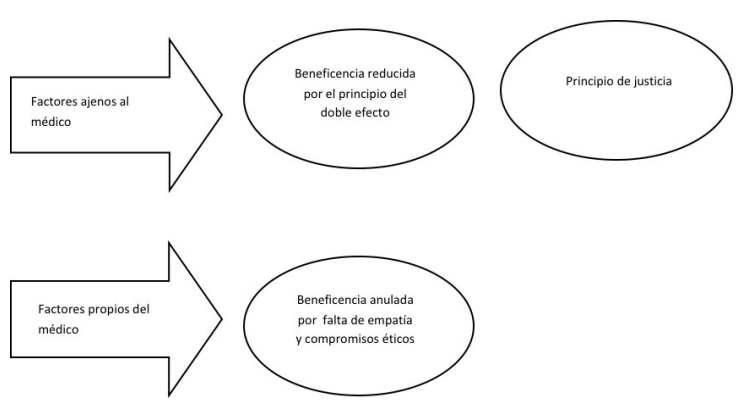

También, es relevante mencionar que, en algunos casos, los médicos actuaron de acuerdo con este principio, apoyando a los pacientes y evitando daños y riesgos, así como reduciendo el malestar y el dolor. No obstante, aún falta que algunos médicos sean entrenados o capacitados para que presten un buen servicio a sus pacientes, para que sean sensibles y empáticos. Esta cuestión resulta difícil en esta época en la que, en ocasiones, el egoísmo predomina sobre el altruismo en los seres humanos que ejercen en las diversas profesiones (17).

\section{Discusión}

Uno de los principios de la bioética principialista es el de beneficencia, referido a todo tipo de acción que pretenda beneficiar a otras personas. Muchos actos de beneficencia no son obligatorios, pero este principio impone a algunos profesionales la obligación de ayudar a otros a promover sus importantes y legítimos intereses. La obligación de conceder beneficios, prevenir y suprimir daños, y sopesar los posibles beneficios frente a los costes y posibles prejuicios de una acción es central para una ética biomédica(3).

Desde la experiencia de algunos de los familiares de los enfermos, se observa que algunos médicos no cumplen con el principio de beneficencia, no se preocupan por dar una buena atención, no canalizan con otros especialistas, no comunican el pronóstico y la asistencia de cuidados paliativos, no prescriben medicamentos adecuados para reducir molestias ocasionadas por los efectos secun- darios de las quimioterapias, no establecen relación con los pacientes.

Algunos médicos quizás suponen que no es importante establecer relación con los pacientes ni proporcionarles información, y prefieren seguir el modelo hegemónico paternalista en el que ellos toman las decisiones.

Pérez y Suazo(18) encontraron que, a pesar del ambiente de diálogo y respeto en relación con la autonomía del paciente y la beneficencia del médico al momento de la toma de decisiones en los tratamientos, el médico tiene la percepción de que ellos los impusieron, y que además les corresponde hacerlo, porque los pacientes no saben de eso. Aquí se refleja un paternalismo duro, ya que el médico está convencido de que ese es su papel y solo un bajo porcentaje (14\%) admite haber dejado la decisión en manos de los pacientes.

De igual forma, Mondragón(19) informa que los médicos, al tratar a pacientes que intentaron suicidarse, actúan de manera paternalista. El médico es la autoridad y da instrucciones de lo que se debe hacer; el paciente que intentó suicidarse es considerado como un sujeto que no tiene juicio ni conciencia, por lo que no se toma en cuenta su autonomía. Sin embargo, a pesar de que las personas puedan tener disminuida su autonomía por la enfermedad, la responsabilidad moral del médico es proporcionar información acerca de su tratamiento.

Otras experiencias de familiares aquí expuestas muestran que algunos médicos sí actuaron de acuerdo con el principio de beneficencia, ayudando a los enfermos. Es importante indicar que estos enfermos fueron dos personas de un nivel socioeconómico medio alto, con estudios de licenciatura o posgrado, un trabajo bien remunerado y fueron atendidos en hospitales privados.

Como se puede apreciar, aunque el principio de beneficencia en la profesión médica implica evitar el daño, asistir a las personas que lo necesiten, brindar un servicio de calidad, con atención respetuosa, evitar los excesos terapéuticos, respetar las creencias y valores y aliviar el daño(20), en ocasiones algunos médicos no logran cumplir con esto. 
Lo preocupante de esta situación es que, en este trabajo, se observa que algunos médicos, cuando trabajan en una institución privada y consiguen mejores remuneraciones por sus servicios, logran cumplir mejor con el principio de beneficencia.

Es claro que en los servicios de salud pública la demanda de pacientes es mayor, por lo que en ocasiones no pueden lograr ser beneficentes, ya sea por falta de empatía, los riesgos de las cirugías, y las limitaciones de tiempo o recursos. No obstante, pese a estos factores, un buen profesional debe actuar lo mejor posible para reducir los daños y evitar el dolor. Se sabe que algunas veces un medicamento puede producir ciertos efectos secundarios en los pacientes, pero si su beneficio es mayor, entonces el médico tendrá que valorar los beneficios y los daños, y sobre todo informar al paciente o familiares.

Es importante que en la comunicación médicopaciente exista un proceso de negociación donde es esencial el diálogo. Este diálogo tiene una triple finalidad: (a) informativa, entendida en el sentido de notificar al paciente sobre las verdaderas condiciones de salud y las opciones terapéuticas disponibles, y sobre las razones éticas o religiosas que sustentan la negativa de realizar una intervención de parte del médico, o de aceptarla por el propio paciente, todo dentro de los límites del secreto profesional; (b) terapéutica, que incluye la eficacia terapéutica que la misma comunicación ejerce; (c) decisoria, porque en la toma de decisiones el diálogo alcanza un significado ético y legal, y es precisamente en este ámbito en el que se plantea el consentimiento informado(21).

Por lo tanto, la beneficencia por parte del médico puede verse afectada por factores externos o factores internos. Los internos pueden ser modificados implementando como parte del curriculum de los estudiantes de medicina valores enfocados en la tolerancia, respeto, altruismo, evitar el daño y sufrimiento. No así los factores externos que dependen más de las instituciones y políticas sanitarias.

\section{Conclusiones}

Las experiencias de los familiares acerca de la beneficencia de los médicos hacia el paciente terminal evidencia que, en ocasiones, estos no actúan de acuerdo con este principio. La falta de información al paciente y su familia, no proporcionar medicamentos o no canalizar con otros especialistas que puedan controlar el dolor o sufrimiento, es un indicativo de que hace falta una mayor capacitación en esta área. Aunque a veces algunos factores, como la carga de trabajo, la presión y la carencia de recursos en los hospitales, impiden que los médicos puedan actuar beneficentemente con los pacientes. Hace falta realizar investigaciones de carácter bioético para que la información obtenida sea utilizada para concientizar a los médicos de su labor en esta área. 


\section{Referencias}

1. Instituto Nacional de Estadística Geografía e Informática. Estadísticas a propósito del día mundial contra el cáncer. México; 2015. Disponible en: http://www.inegi.org.mx/inegi/default.aspx?c=274

2. Cataldi A. Manual de ética médica. Ciudad de Buenos Aires: Universidad; 2003.

3. Beauchamp LT, Childress FJ. Principios de Ética Biomedica. Espańa: Masson; 2002.

4. Rivero SO, Paredes SR. Ética en el ejercicio de la medicina. México: Panamericana; 2006.

5. Wulff RH, Rosenberg R. Introducción a la filosofía de la medicina. Madrid: Triacastela; 2002.

6. Agirre-Gas H. Principios éticos de la práctica médica. Cirugía y cirujanos 2004; 72(6): 503-510.

7. Van Manen M. Researching lived experience: Human science for an action sensitive pedagogy. London, Ontario, Canada: Althouse Press; 1999.

8. Dickinson RA. The family/practitioner relationship in the context of chronic childhood illness. Thesis. Degree Doctor of Philosophy. Auckland University of Technology; 2004.

9. Lopez KA, Willis DG. Descriptive versus interpretive phenomenology: their contributions to nursing knowledge. Qualitative Health Research 2004; 14 (5): 726-735.

10. Watt SJ, Lynn WR. A journey within a journey: the lived experience of parents who do not live near their child's tertiary cancer center. Journal of Pediatric Oncology Nursing 2011; 28(4): 231-243.

11. Broussard BB. Women's experiences of bulimia nervosa. Journal of Advanced Nursing 2005; 49(1): 43-50.

12. Edvardsson JD, Sandman P, Rasmussen BH. Meanings of living touch in the care of older patients: becoming a valuable person and professional. Journal of Clinical Nursing 2003; 12: 601-609.

13. Todres L, Fulbrook P, Albarran J. On the receiving end: a hermeneutic phenomenological analysis of a patient's struggle to cope while going through intensive care. Nursing in Critical Care 2003; 5(6): 284.

14. Svedlund, M, Danielson E, Norberg A. Women's narratives during the acute phase of their myocardial infarction. Journal of Advanced Nursing 1994: 35(2): 197-205.

15. Gullickson, C. My death nearing is future: a Heideggerian hermeneutical analysis of the lived experience of persons with chronic illness. Journal of Advanced Nursing 1993; 18: 1386-1392.

16. Martínez-Salgado C. El muestreo en investigación cualitativa. Principios básicos y algunas controversias. Ciênc. saúde coletiva 2012: 17(3).

17. Ruiz, P, Sánchez, M. (2006). Profesión y profesionalismo en medicina. Seminario "El ejercicio actual de la medicina". Disponible en: http://www.facmed.unam.mx/sms/seam2k1/2006/nov_01_ponencia.html

18. Pérez B, Suazo M. El consentimiento informado, autonomía y beneficencia en la consulta hospitalaria de la ciudad de Santo Domingo (II). Ciencia y Sociedad 2010; XXXV(1): 7-20.

19. Mondragón L, Monroy Z, Ito E, Medina-Mora E. Disyuntivas en las concepciones sobre Autonomía y Beneficencia que afectan la terapéutica del intento suicida. Acta Bioethica 2010; 16(1): 77-86.

20. Sánchez Z, Marrero N, Becerra O, Herrera D, Alvarado J. Los principios bioéticos en la atención primaria de salud. Apuntes para una reflexión. Rev Cubana Med Gen Integr 2003; 19(5).

21. Lee G, Sotelo G, Casa O. La objeción de conciencia en la práctica del médico. Revista de la Facultad de Medicina de la UNAM 2006; 49(3): 121-125.

Recibido: 11 de noviembre de 2016

Aceptado: 3 de julio de 2017 